\title{
MicroRNA-17-92 regulates myoblast proliferation and differentiation by targeting the ENH1/ld1 signaling axis
}

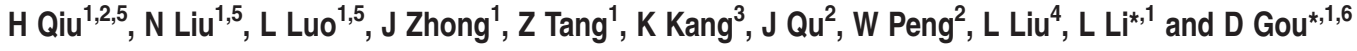

Myogenesis is an important biological process that occurs during both skeletal muscle regeneration and postnatal growth. Growing evidence points to the critical role of microRNAs (miRNAs) in myogenesis. Our analysis of miRNA expression patterns reveal that miRNAs of miR-17-92 cluster are dramatically downregulated in $\mathrm{C}_{2} \mathrm{C} 12$ cells after myogenesis stimulation, are strongly induced in mouse skeletal muscle after injury and decrease steadily thereafter and are downregulated with age in skeletal muscle during mouse and porcine postnatal growth. However, their roles in muscle developmental processes remain elusive. We show that the miR-17-92 cluster promotes mouse myoblast proliferation but inhibits myotube formation. miR-17, -20a and -92a target the actin-associated protein enigma homolog 1 (ENH1). The silencing of ENH1 increased the nuclear accumulation of the inhibitor of differentiation 1 (Id1) and represses myogenic differentiation. Furthermore, the injection of adenovirus expressing miR-20a into the tibialia anterior muscle downregulates ENH1 and delays regeneration. In addition, the downregulation of miR-17-92 during myogenesis is transcriptionally regulated by E2F1. Overall, our results reveal a E2F1/miR-17-92/ENH1/ld1 regulatory axis during myogenesis.

Cell Death and Differentiation (2016) 23, 1658-1669; doi:10.1038/cdd.2016.56; published online 17 June 2016

Myogenesis is an important event during postnatal muscle growth and regeneration. ${ }^{1,2}$ It is a multistep process during which myoblasts proliferate, withdraw from the cell cycle, differentiate into myocytes, fuse into multinucleated myotubes with centralized nuclei, and undergo further maturation. ${ }^{3}$ Myogenic regulatory factors (MRFs) including MyoD, Myf5, myogenin and MRF4 work in conjunction with $E$ proteins to activate muscle gene expression. ${ }^{4,5}$ On the other hand, one of the master myogenesis inhibitors, inhibitor of differentiation (Id), competitively binds to MRF and/or $E$ proteins and subsequently inhibits myogenic differentiation. ${ }^{6}$ However, the induction of Id1 reverses the neonatal lethality of transgenic mice overexpressing myogenin in skeletal muscle. ${ }^{7}$ It is clear that investigating the mechanism by which muscle cells modulate the interaction between myogenic factors and Id is required for a better understanding of muscle growth and regeneration.

Increasing evidence suggests that microRNAs (miRNAs) are involved in skeletal muscle development and regeneration. To date, $\sim 30$ miRNAs have been experimentally identified as myogenesis-associated miRNAs. ${ }^{8-10}$ These miRNAs, which have been discovered primarily from studies using the in vitro model of skeletal muscle myogenesis, mouse C2C12 cells, play important roles in skeletal muscle regeneration and growth. The loss of miR-206 or the knockdown of miR-26a delays the normal kinetics of muscle regeneration in mice. ${ }^{11,12}$ The double knockout of miR-208b and miR-499 causes a dramatic loss of type I fibers in the soleus muscle in mice. ${ }^{13}$ However, it is clear that there are still many myogenesisassociated miRNAs that have yet to be discovered.

In this study, to identify additional potential myogenic miRNAs, we analyzed the miRNA expression profiles of proliferating and differentiating $\mathrm{C} 2 \mathrm{C} 12$ cells using a novel method of S-Poly(T) Plus real-time PCR. ${ }^{14,15}$ This method is one of the most sensitive and accurate methods for the quantification of miRNAs. Of the 720 detected miRNAs, 55 were differently expressed by at least fourfold. Among these, three members of the miR-17-92 cluster (miR-17, -20a and -92a) were highly expressed in the proliferating $\mathrm{C} 2 \mathrm{C} 12$ myoblasts and significantly decreased in the differentiating C2C12 cells. Although miR-17-92 has been well-studied for its role in tumorigenesis, ${ }^{16-19}$ its role in skeletal muscle myogenesis remains undetermined. Because the proliferation of skeletal muscle cells is an initial step in muscle growth and regeneration, ${ }^{20}$ it is important to understand the regulatory network through which miR-17-92 controls the steps of myogenesis. We thus further demonstrated the expression pattern of the miR-17-92 cluster during myogenesis, muscle regeneration and postnatal growth. In vitro and in vivo studies established that miR-17-92 plays an important role in skeletal

${ }^{1}$ Shenzhen Key Laboratory of Microbial Genetic Engineering, College of Life Sciences, Shenzhen University, Shenzhen, Guangdong 518060, China; ${ }^{2}$ Key Laboratory of Optoelectronic Devices and Systems of Ministry of Education and Guangdong Province, College of Optoelectronic Engineering, Shenzhen University, Shenzhen, Guangdong 518060, China; ${ }^{3}$ Department of Biochemistry and Molecular Biology, School of Basic Medical Sciences, Shenzhen University Health Science Center, Shenzhen, Guangdong 518000, China and ${ }^{4}$ Department of Physiological Sciences, Oklahoma State University, Stillwater, OK 74078, USA

${ }^{*}$ Corresponding author: D Gou or L Li, Shenzhen Key Laboratory of Microbial Genetic Engineering, College of Life Sciences, Shenzhen University, Shenzhen, Guangdong 518060, China. Tel: +86 0755 26527848; Fax: +86 0755 26527848; E-mail: dmgou@szu.edu.cn or ylili@ szu.edu.cn

${ }^{5}$ These authors contributed equally to this work.

${ }^{6}$ Current address: Shenzhen Key Laboratory of Microbial Genetic Engineering, College of Life Sciences, Shenzhen University, Shenzhen, Guangdong 518060, China. Abbreviations: CTX, Cardiotoxin; DM, differentiation medium; EdU, ethynyl-2'-deoxyuridine; ENH1, enigma homolog 1; GM, growth medium; Id, inhibitor of differentiation; MRF, myogenic regulatory factor

Received 08.12.15; revised 19.5.15; accepted 20.5.16; Edited by J-C Marine; published online 17.6.2016 
muscle cell myogenesis by downregulating enigma homolog 1 $(\mathrm{ENH} 1)$, by directly targeting its $3^{\prime}$ untranslated regions (3'UTR). Finally, we showed that E2F1 transcriptionally regulates miR-17-92 during muscle myogenesis. Therefore, our study not only elucidates the roles of miR-17-92 in skeletal muscle differentiation and development, it reveals the mechanism which miRNA regulates myogenesis by modulating a well-established inhibitor of myogenic differentiation.

\section{Results}

miR-17-92 was downregulated during myoblast myogenesis, skeletal muscle regeneration and postnatal growth. To explore the potential miRNAs that are involved in muscle development, we first analyzed the expression patterns of miRNAs during myogenesis. We used the mouse $\mathrm{C} 2 \mathrm{C} 12$ myoblast, an immortal skeletal muscle cell line that has been proven to be an ideal cell model for the in vitro study of myoblast myogenesis. ${ }^{21,22}$ The $\mathrm{C} 2 \mathrm{C} 12$ myoblasts proliferated in growth medium (GM) and were induced to differentiate into myotubes with serum depletion in differentiation medium (DM) (Supplementary Figures S1a and b). A total of 720 miRNAs were quantified using the S-poly (T) plus miRNA quantitative real-time PCR (qRT-PCR) method (Figures 1a and $b$ ). After two rounds of screening, 55 miRNAs were found to be differentially expressed by more than fourfold between the myoblasts (cells in GM) and the myotubes (cells in DM4). A total of 16 of these 55 miRNAs have been reported to be myogenesis-associated miRNAs. ${ }^{9,23,24}$ For the 39 novel candidates, 30 miRNAs were found to be upregulated and only 9 were found to be downregulated in the myotubes (Figure 1a). The downregulated miRNAs include three members of the miR-17-92 cluster (miR-17, -20a and -92a, Figure 1b). Further investigation at different differentiation time points confirmed that the miR-17-92 cluster (miR-17, $-18 a,-19 a,-19 b,-20 a$ and $-92 a)$ is expressed at high levels in the proliferating myoblasts but at low levels during differentiation (Figure 1c). We next analyzed the expression of this miRNA cluster during skeletal muscle regeneration using the Cardiotoxin (CTX) induced skeletal muscle damage and regeneration model, which mimics in vivo muscle differentiation. As anticipated, following the CTX injection into the mouse tibialis anterior muscle, the activated muscle satellite cells proliferate (1-3 days) and then differentiate to replenish the damaged myofibers (3-10 days), as confirmed by hematoxylin and eosin (H\&E) staining and the mRNA expression patterns of $\operatorname{Pax} 7$ and $M y o D$ (Supplementary Figures S2 and S3). We found that miR-17-92 was highly expressed 3 days post injury and subsequently decreased in expression thereafter (Figure 1d). The miR-17-92 cluster is involved in organ development. ${ }^{17,25}$ However, little is known about its temporal expression pattern during skeletal muscle development. The age-dependent decrease in the expression of miR-17-92 was found in the porcine longissimus dorsi muscles and the mouse hind leg muscles during postnatal development (Figure 1e and f). Taken together, these data indicate that the downregulation of miR-17-92 is associated with myoblast myogenesis and skeletal muscle development and regeneration.
miR-17-92 promotes $\mathrm{C} 2 \mathrm{C} 12$ myoblast proliferation but prevents differentiation. To study the functions of miR-17-92, we first investigated whether miR-17-92 affects myoblast proliferation. We infected $\mathrm{C} 2 \mathrm{C} 12$ cells with a lentivirus individually overexpressing or inhibiting three major miRNAs of this cluster (miR-17, -20a and -92a). Based on the ethynyl2 -deoxyuridine (EdU) incorporation assay, we observed that the overexpression of miR-17, $-20 \mathrm{a}$ or $-92 \mathrm{a}$ significantly increased cell proliferation (1.37-fold, 1.44-fold and 1.43-fold, respectively, $P<0.01$ ) compared with the control virus (miR-NC) (Figure $2 \mathrm{a}$ and Supplementary Figure S4a). In contrast, the knockdown of miR-17, -20a or -92a caused the opposite effect (Figure $2 \mathrm{~b}$ and Supplementary Figure S4b). These data show that miR-17, $-20 a$ and $-92 a$ have proproliferative effects.

Next, we investigated the role of the miR-17-92 cluster on C2C12 differentiation. First, we performed dual-luciferase reporter assays by co-transfecting $\mathrm{C} 2 \mathrm{C} 12$ myoblasts with plasmids overexpressing miR-17, $-20 a$ or $-92 a$ and the myogenin promoter-driven Firefly luciferase. The overexpression of miR-17, -20 a or $-92 a$ reduced the luciferase activity (Figure 2c), implying that these miRNAs affect myogenic differentiation. The $\mathrm{C} 2 \mathrm{C} 12$ myoblasts were then transfected with the miR-17, -20a or -92a mimics, or the control (miR-NC) and transferred to DM. As shown in Figure 2d, the overexpression of miR-17, -20 a or $-92 a$ dramatically blocked myotube formation when compared with the miR-NC group. Their inhibitory effects on muscle differentiation were further confirmed by western blot as indicated by a decrease in myogenin protein expression levels in $\mathrm{C} 2 \mathrm{C} 12$ cells or in primary mouse myoblasts (Figure $2 \mathrm{e}$ and Supplementary Figure S5). Collectively, these results indicate that miR-17, $-20 \mathrm{a}$ and $-92 \mathrm{a}$ promote proliferation but have inhibitory effects on myoblasts differentiation.

ENH1 is a common target of miR-17, $-20 a$ and $-92 a$ and represses myogenesis. We next investigated the mechanism through which miR-17-92 regulates myogenesis by searching for miRNA targets that might mediate its effects. According to TargetScan 6.2 (Cambridge, MA, USA) and FINDTAR3 (Shenzhen, China), ENH1 is a common predicted target of miR-17, $-20 a$ and $-92 a$. ENH1 revealed an inverse relation with $\mathrm{miR}-17,-20 \mathrm{a}$ or $-92 \mathrm{a}$ expression not only during C2C12 myogenesis but also during skeletal muscle regeneration (Figures $3 a$ and $b$ and Supplementary Figure S6), indicates that ENH1 is a potential target of miR-17, -20a and $-92 a$ in regulating muscle cell proliferation and differentiation. ENH1 has one binding site (2234-2257) in its $3^{\prime}$ UTR for miR-17 and -20a and another binding site (2791-2811) for miR-92a (Figure 3c). As shown in Figure 3d, the overexpression of miR-17, -20 a or $-92 a$ significantly repressed the luciferase activities of the wild-type ENH1 3'UTR reporter (0.58-, 0.73- and 0.60- fold, $P<0.001$, respectively), whereas these repressions were completely abolished when their corresponding binding sites were mutated. To further validate our results, the $\mathrm{C} 2 \mathrm{C} 12$ cells were transfected with the miR-17, -20a, -92a mimics or the control mimic (miR-NC). Consistent with the above $3^{\prime}$ UTR dual-luciferase assay, the overexpression of $\mathrm{miR}-17,-20 \mathrm{a}$ or $-92 \mathrm{a}$ resulted in a decrease in the expression of $\mathrm{ENH}_{1}$ at the protein level 
a

C2C12 myoblasts (GM) and differentiated myotubes (DM4)

$\downarrow$

Exploratory screening of 720 miRNAs [S-Poly(T) Plus qPCR ]

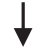

Analysis of miRNAs differentially expressed

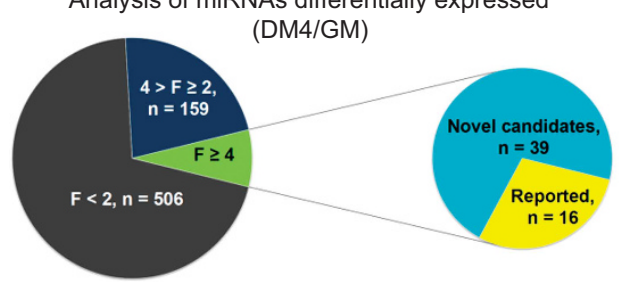

Analysis of expression patterns of novel candidate miRNAs

(9 downregulated and 30 upregulated)

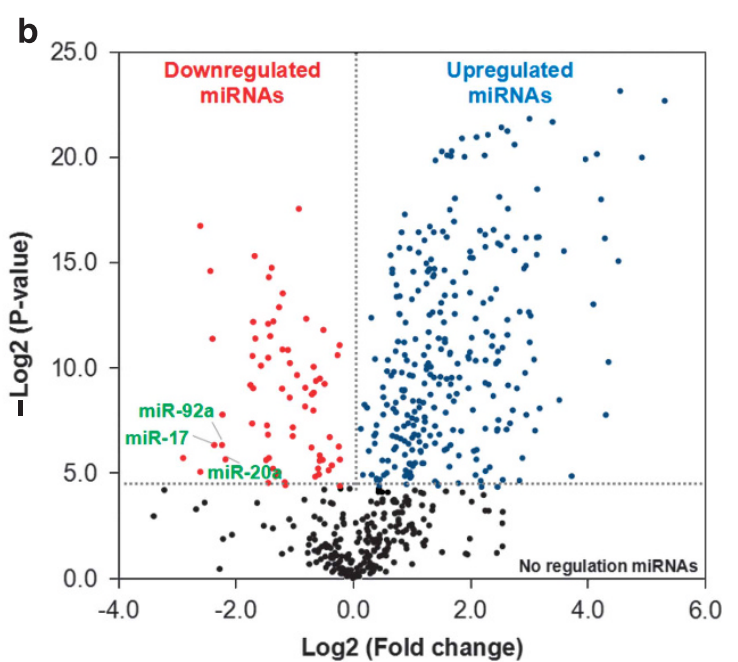

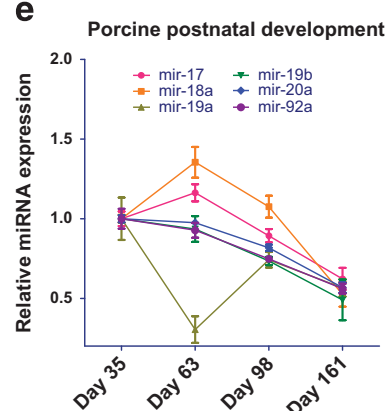

f

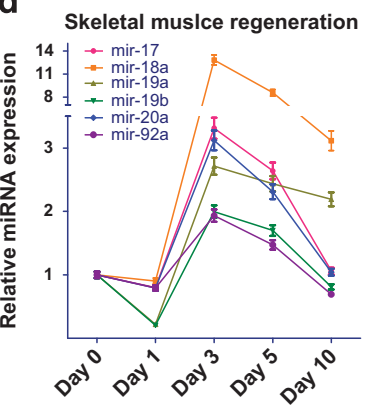

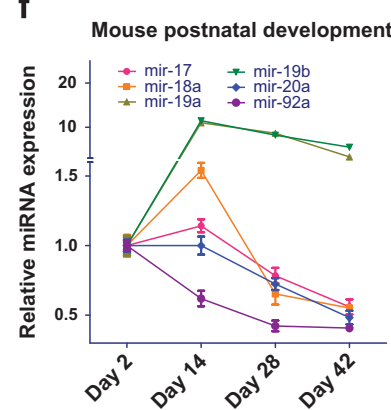

Figure $1 \mathrm{miR}-17-92$ cluster is downregulated during skeletal muscle differentiation, regeneration and development. (a) Workflow of the identification of myogenesisassociated miRNAs. (b) Volcano plot showing the differentially expressed miRNAs in differentiated C2C12 myotubes compared with myoblasts. The negative Log2-adjusted $P$-values ( $y$-axis) are plotted against the Log2 fold changes in expression ( $x$-axis). The horizontal dashed line indicates the threshold for significance $(P<0.05)$ and the vertical dashed line indicates the upregulated (right side) and downregulated (left side) miRNAs. miR-17,-20a and -92a are indicated. (c) miR-17-92 is downregulated during myogenesis. $\mathrm{C} 2 \mathrm{C} 12$ mouse myoblasts were cultured in growth medium (GM) and then switched to differentiation medium (DM) for 1-7 days. Quantitative RT-PCR was performed to analyze the expression level of miR-17, -18a, -19a, -20a and -92a. The data were normalized to SNORNA234. miR-17-92 expression in myoblasts cultured in DM was set to 1.0. The error bars depict the means \pm S.D. of three independent cell samples. (d) miR-17-92 is upregulated in skeletal muscle on days 1-3 and downregulated on days 5-10 post-CTX injury. miR-17-92 expression in skeletal muscle before the CTX injection was set to 1.0. The error bars depict the means \pm S.D. of the samples with six mice in each group. The rest is as in c. (e) miR-17-92 expression in porcine skeletal muscle during post-natal development. Quantitative RT-PCR validated the level of miR-17-92 in porcine longissimus dorsi muscles on postnatal days $35,63,98$ and 161. The Data were normalized to SNORAN202. miR-17-92 expression on postnatal day 35 was set to 1.0. The error bars depict the means \pm S.D. of the samples with six pigs in each group. $(\mathrm{f})$ miR-17-92 expression in mouse skeletal muscle during postnatal development. qRT-PCR validated the level of miR-17-92 in mouse hind legs on days 2, 14, 28 and 42. miR-17-92 expression on day 2 was set to 1.0. The rest is as in $\mathbf{d}$

(0.59-fold, $\quad P<0.05, \quad 0.36$-fold, $\quad P<0.01$ and 0.77 -fold, $P<0.05$, respectively, Figure $3 \mathrm{e})$. In contrast to miRNA overexpression, transfection of miR-17, $-20 \mathrm{a}$ or $-92 \mathrm{a}$ inhibitors in the $\mathrm{C} 2 \mathrm{C} 12$ cells resulted in an increase of $\mathrm{ENH} 1$ protein about 1.5-fold $(P<0.01)$ (Figure 3f). Based on these data, we conclude that ENH1 is a direct target of miR-17, $-20 a$ and $-92 a$ in myoblasts.

To further investigate the role of the $\mathrm{ENH} 1$ on $\mathrm{C} 2 \mathrm{C} 12$ myogenesis, we constructed the si-ENH1 vector and confirmed its efficiency by western blot (Figure $3 \mathrm{~g}$ ). We subsequently transferred the si-ENH1 myoblasts to DM for 4 days. Consistent with the inhibitory effect of miR-17-92 on muscle differentiation, the silencing of ENH1 dramatically blocked myotube formation and repressed myogenin protein expression (Figures $3 \mathrm{~h}$ and i). Again, consistent with the proproliferative effects of miR-17, $-20 a$ and $-92 a$, the silencing of ENH1 significantly increased the cell proliferation rate (Figure 3j and Supplementary Figure S7). Conversely, the forced expression of ENH1 in $\mathrm{C} 2 \mathrm{C} 12$ myoblasts reduced proliferation (Figure 3k and Supplementary Figure S7). These results show that miR-17, -20 a and -92a repress ENH1 expression, which in turn contributes to their functions of promoting muscle proliferation and preventing differentiation.

miR-17-92 and ENH1 modulate the abundance of Id1. The process of myogenic differentiation is regulated by at least three protein families including $E$ protein, muscle regulatory factor and Id proteins. ${ }^{6}$ Ids (Id1-4) are a group of ubiquitous nuclear proteins that bind to MRF and $E$ protein, which prevents the heterodimerization of MRF and $E$ protein in nucleus and subsequently inhibits myogenic differentiation. ${ }^{26,27}$ ENH proteins are well-established regulators of the subcellular localization of helix-loop-helix proteins including Ids. ${ }^{28}$ In $\mathrm{C} 2 \mathrm{C} 12$ myoblasts, Id1 mRNA is present at higher levels than Id2, Id 3 or Id 4 in myoblasts (Supplementary Figure S8). We thus investigated the regulatory 

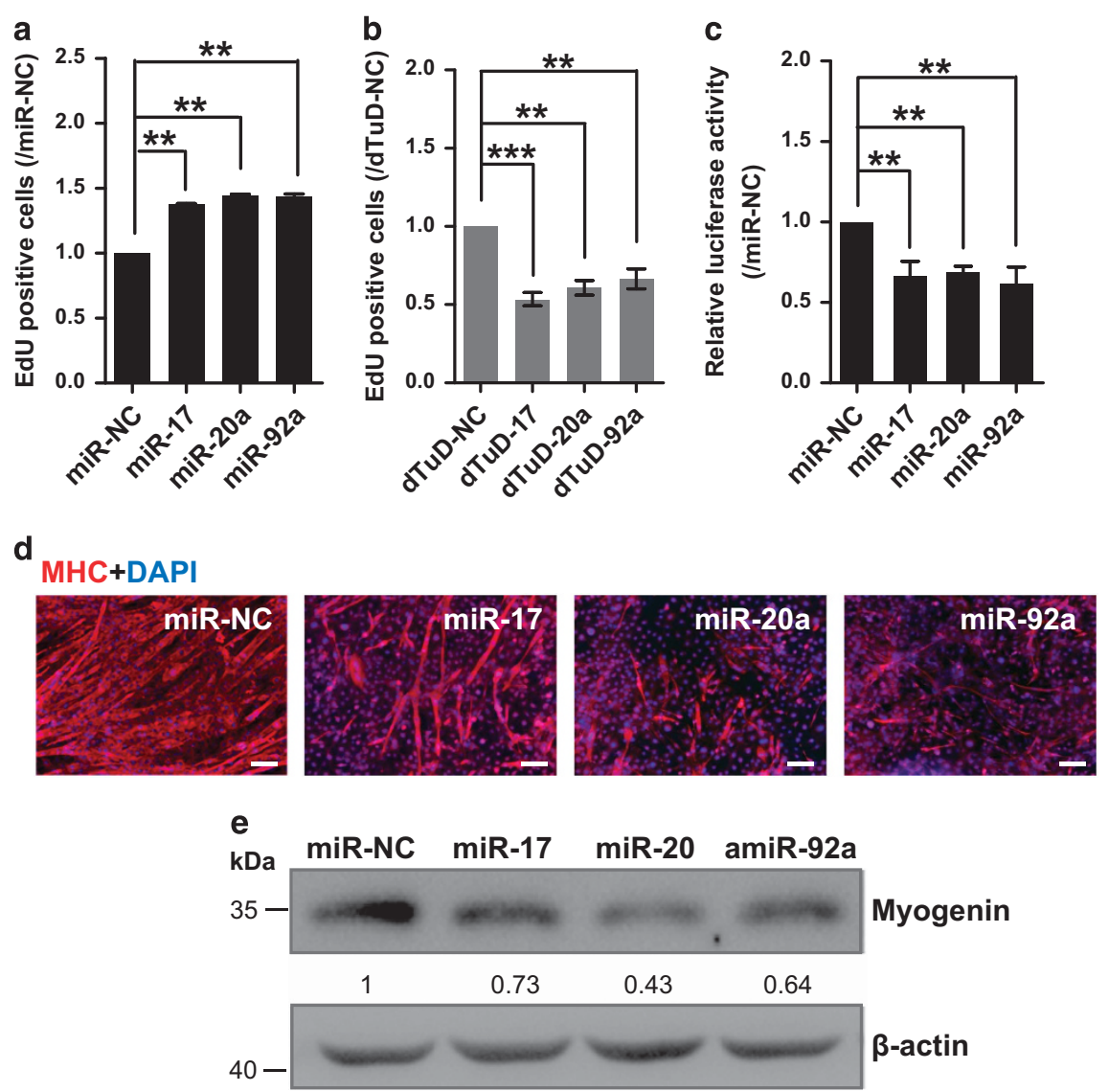

Figure 2 miR-17-92 enhances myoblast proliferation and represses differentiation. (a) Overexpression of miR-17, -20a or -92a enhances the rate of myoblast proliferation. The cell proliferation analysis was performed by EdU incorporation of myoblasts infected with lentiviruses expressing miR-17,-20a or -92a. The cell number of the negative control (miR-NC) was set to 1.0. The error bars depict the means \pm S.D. of three replicates. ${ }^{* *} P<0.01$. (b) Inhibition of miR-17, -20 a or $-92 a$ reduces the rate of myoblast proliferation. $\mathrm{C} 2 \mathrm{C} 12$ mouse myoblasts were lentivirally infected with the miRNA inhibitors dTuDs targeting miR-17, -20 a or $-92 a$ (dTuD-17, -20 a or $-92 a$ ) or dTuD-NC. ${ }^{* *} P<0.01$ and ${ }^{* \star *} P<0.001$. The rest is as in a. (c) Myogenin promoter luciferase assay demonstrates that miR-17, $-20 \mathrm{a}$ and $-92 \mathrm{a}$ reduce the activity of myogenin promoter. C2C12 myoblasts were co-transfected with the pSV40-R.Luc vector, the myogenin promoter luciferase reporter and the vector expressing miR-17, $-20 \mathrm{a},-92 \mathrm{a}$ or NC, and the cells were transferred to DM for 3 days. The luciferase activity of the myoblast transfected with miR-NC was designated as 1.0. The error bars depict the means \pm S.D. of three replicates. ${ }^{* *} P<0.01$. (d) The miR-17, -20a or -92a mimics repress $\mathrm{C} 2 \mathrm{C} 12$ myoblast differentiation. $\mathrm{C} 2 \mathrm{C} 12$ myoblasts were transfected with the miRNA mimics as indicated, and the cells were transferred to DM and then stained for MHC (red) and DAPI (blue) at DM4. Scale bar, $100 \mu \mathrm{m}$. (e) Western blot shows that the overexpression of miR-17, -20a or -92a reduces myogenin protein expression. $\mathrm{C} 2 \mathrm{C} 12$ myoblasts were transfected with miRNA mimics or NC and transferred to DM for 4 days. Beta-actin served as the loading control. The data are presented as the means of the samples from three different cell samples

axis of miR-17/20a/92a-ENH1-ld1 in muscle differentiation. Id1 protein was downregulated during differentiation (Figure 4a), which displayed an inverse correlation with ENH1 (Supplementary Figure S9a). This inverse correlation was found only in the late phage of muscle regeneration (Supplementary Figures S9b and c). On the other hand, ENH1 has been reported to promote the cytoplasmic sequestration of Id2 during neuronal cell differentiation. ${ }^{28}$ We thus investigated whether ENH1 regulates the Id1 during myogenic differentiation.

We found that Id1 was less abundant in the cytoplasm as well as in the nucleus at DM4 (Figure 4b). Consistent with the study of Sun et al..$^{29}$ that Id1 localizes in the nucleus of proliferating $\mathrm{C}_{2} \mathrm{C} 12$ myoblasts while in the cytoplasm of mature myotubes, little exogenous Id1 was found in the nucleus of the differentiating $\mathrm{C} 2 \mathrm{C} 12$ cells (Figure 4c). However, the exogenous Id1 was mostly localized in the nucleus of
si-ENH1 myoblasts (Figure 4d). Moreover, the silencing of ENH1 caused an increase of endogenous Id 1 in the whole cell (1.53-fold, $P<0.05)$ and in the nucleus (1.32-fold, $P<0.05)$ (Figure 4e). These results show that the abundance of Id1 in the nucleus depended on the ENH1 expression level.

Consistent with the effects of si-ENH1, the overexpression of miR-17, $-20 a$ or $-92 a$ resulted in more exogenous Id1 accumulated in the nucleus (Figure 4f). Further analysis shows that the overexpression of miRNA reduced ENH1 protein expression while increased the abundance of endogenous Id1 in the cells at GM and DM4 (Figure 4g). Moreover, a mixture of miR-17, $-20 \mathrm{a}$ and $-92 \mathrm{a}$ mimics increased the Id1 protein level in the cytoplasm (1.30-fold, $P<0.05)$ but not in the nucleus (1.1-fold, $P>0.05$ ) (Figure 4h). A further qPCR showed that the overexpression of miR-17, $-20 \mathrm{a}$ and $-92 \mathrm{a}$ increased the transcriptional level of $\mathrm{Id} 1$ in the $\mathrm{C} 2 \mathrm{C} 12$ cells (Figure 4i). Therefore, although ENH1 could reduce the 
a

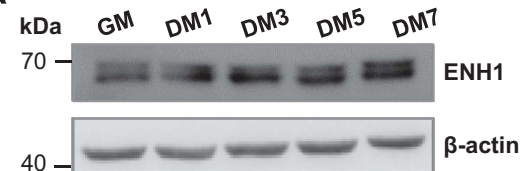

e

kDa miR-NC miR-17 miR-20a miR-92a

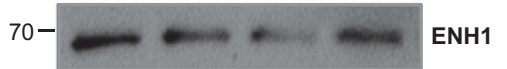

$\begin{array}{llll}1 & 0.59 & 0.36 & 0.77\end{array}$

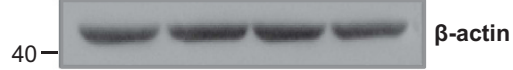

f

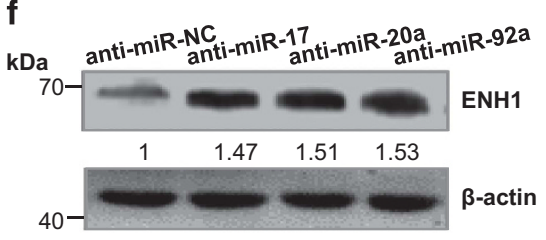

g

$\mathrm{g}$

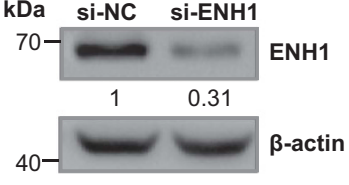

i

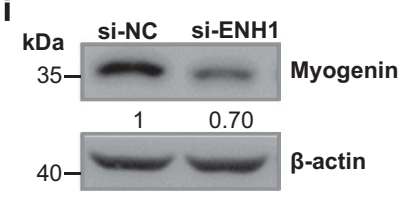

b

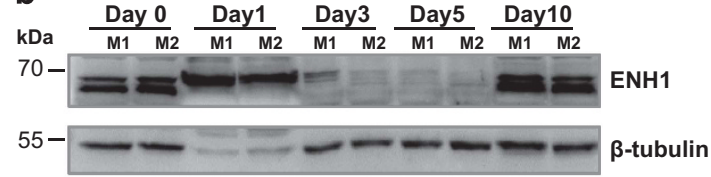

C

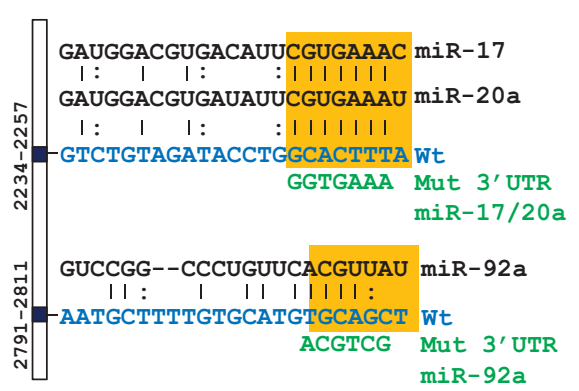

$\operatorname{miR}-92 a$
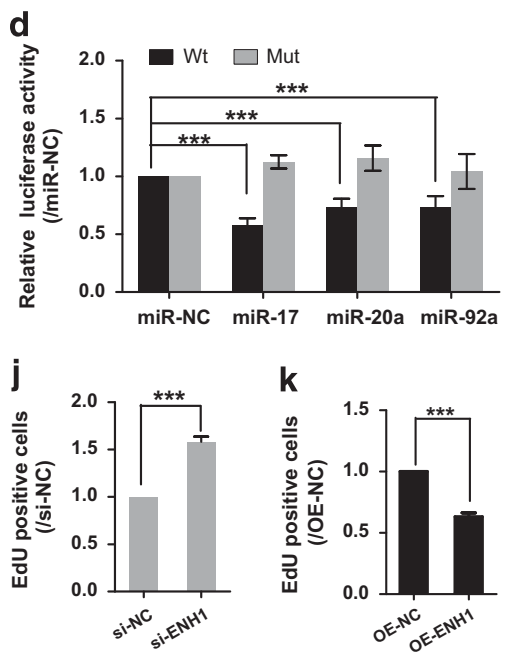

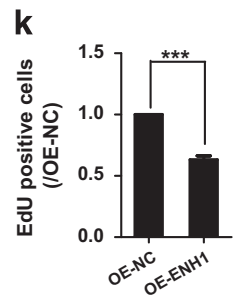

Figure 3 miR-17, -20a and -92a downregulate ENH1 protein expression by directly targeting its $3^{\prime}$ UTR and ENH1 promotes myogenic differentiation but inhibits cell proliferation. (a) ENH1 protein expression during $\mathrm{C} 2 \mathrm{C} 12$ myoblast myogenesis. $\mathrm{C} 2 \mathrm{C} 12$ mouse myoblasts were cultured in growth medium (GM) and then switched to differentiation medium (DM) for 1-7 days. Beta-actin served as the loading control. (b) ENH1 protein expression in skeletal muscle during regeneration after CTX injury. Betatubulin served as the loading control. M1 and M2, mouse 1 and mouse 2. (c) Schematic illustration of the predicted binding sites for miR-17 and -20a (2234-2257) and for miR-92a (2791-2811) in the $3^{\prime}$ UTR of ENH1. The wild -type miRNA-binding sites (orange) were modified to complementary sequences (green) to construct the mutated ENH1 $3^{\prime} \mathrm{UTR}$. (d) ENH1 3'UTR or 3'UTR Mut constructs were co-transfected with the miR-17,-20a or -92a mimics into $293 \mathrm{~A}$ cells. The data were normalized to the Renilla luciferase activity. The luciferase activity in cells transfected with miR-NC was set to 1.0. The error bars depict the means \pm S.D. of three measurements. ${ }^{* * *} P<0.001$. (e) Western blot analysis of ENH1 protein in the $\mathrm{C} 2 \mathrm{C} 12$ myoblasts transfected with miR-17, -20a, -92a or NC mimics and (f) with miR-17, -20a, -92a or NC inhibitors. Beta-actin served as the loading control. The data are presented as the means of the samples from three different cell samples. (g) Western blot confirms the efficiency of si-ENH1 on ENH1 protein expression in myoblasts. The rest is as in e. ( $\mathbf{h}$ and i) si-ENH1 blocks $\mathrm{C} 2 \mathrm{C} 12$ myoblast differentiation. $\mathrm{C} 2 \mathrm{C} 12$ myoblasts were transfected with si-ENH1 or si-NC and stimulated to differentiate. Immunostaining of $\mathrm{MHC}(\mathrm{h})$ and western blot (i) of myogenin showing that si-ENH1 resulted in smaller myotubes and a decrease of myogenin protein at DM4. Scale bar, $200 \mu \mathrm{m}$. The rest is as in $\mathbf{e}$. ( $\mathbf{j}$ and $\mathbf{k}$ ) ENH1 modulates cell proliferation. $\mathrm{C} 2 \mathrm{C} 12$ myoblasts were lentivirally transfected with si-ENH1 or infected with vectors expressing ENH1 and maintained in GM. The EdU incorporation assay was performed for the analysis of cell proliferation. The silencing of ENH1 (j) enhanced EdU incorporation compared with the control (si-NC), whereas the overexpression of ENH1 (k) inhibited cell proliferation. The error bars depict the means \pm S.D. of three measurements. ${ }^{* * \star} P<0.001$

nuclear Id1 in myoblasts, miR-17-92 modulates the protein level of Id1 mostly by other regulatory axis rather than $\mathrm{ENH} 1$ mediated pathway.

miR-20a promotes proliferation but prevents differentiation of regenerating skeletal muscle. Considering the fact that miR-20a effectively inhibits ENH1 expression in vitro (Figure 3e), we injected an adenovirus overexpressing miR-20a or its control (Ad-miR-NC) into the tibialis anterior muscles of mice and then evaluated the miR-20a-mediated regulation of muscle proliferation and differentiation using the CTX-induced skeletal muscle damage and regeneration model. Ten days after the Ad-miR-20a injection, miR-20a was upregulated (1.74-fold, $P<0.05$ ) in the muscle (Figure 5a), and the miR-20a target protein ENH1 was dramatically downregulated in the Ad-miR-20a mice (Figure 5b). As previously described, miR-17-92 was upregulated 1-3 days post injury, which is the active phase of muscle proliferation (Figure 1d). We investigated whether the overexpression of miR-20a affects muscle cell proliferation. The results of $\mathrm{H} \& \mathrm{E}$ staining of muscle cross-sections show that Ad-miR-20a induced an accumulation of mononucleated cells in the 


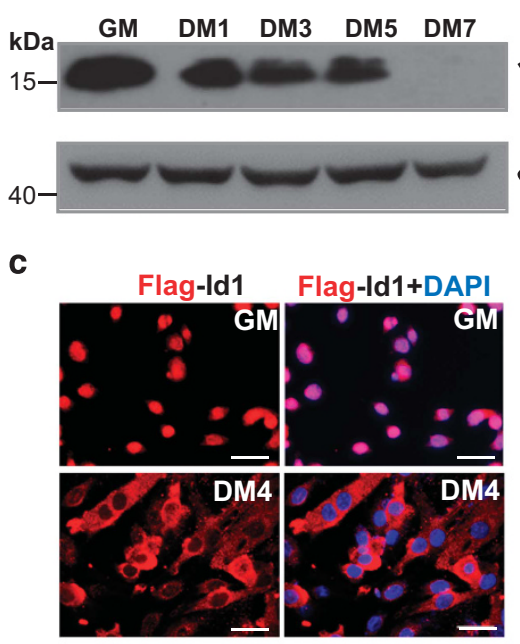

d
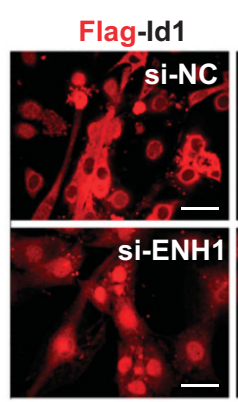

Flag-Id1+DAPI

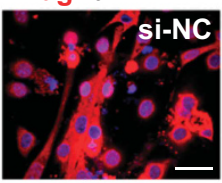

f

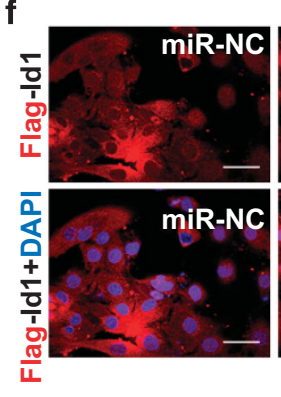

b
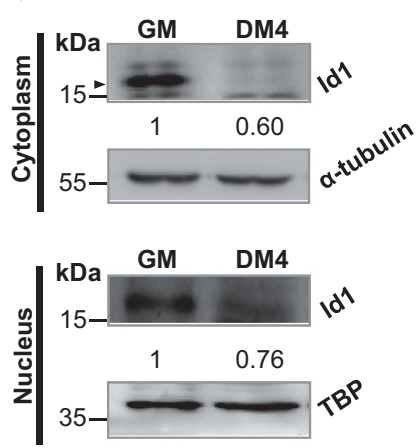

h
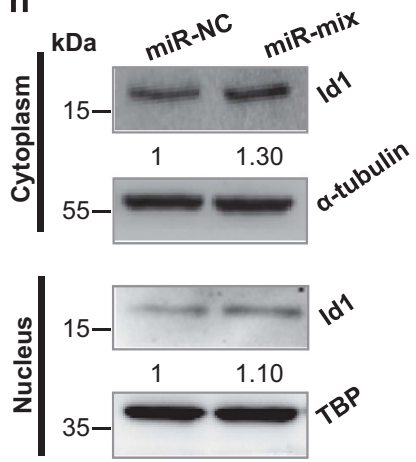
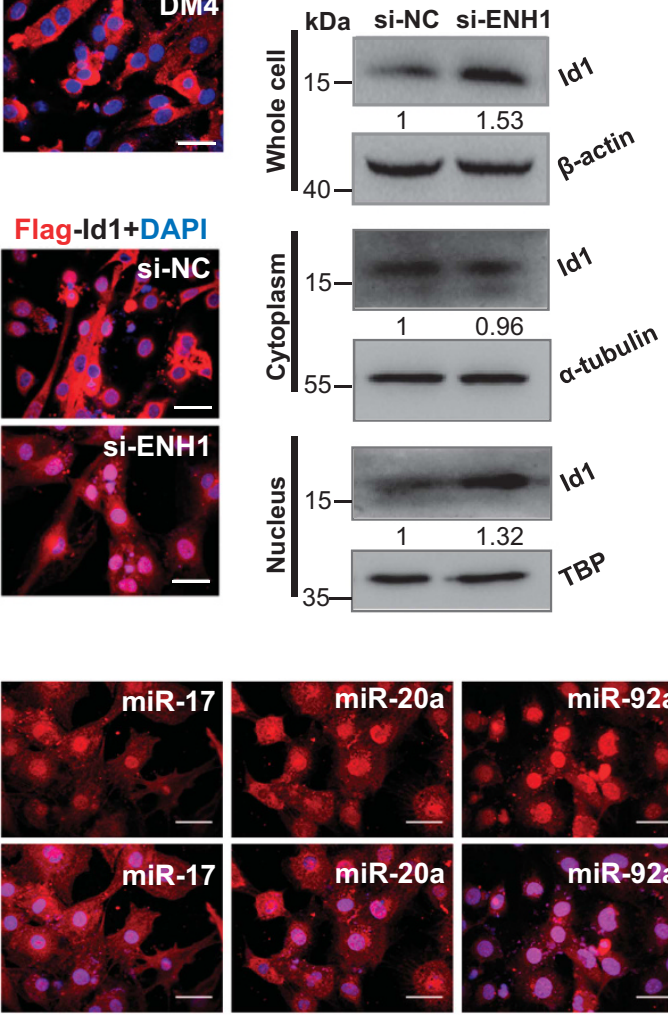

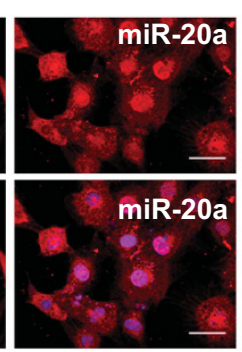

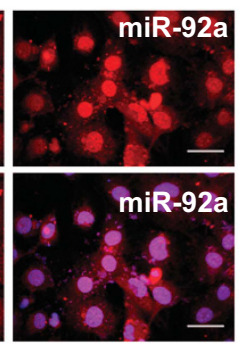

9
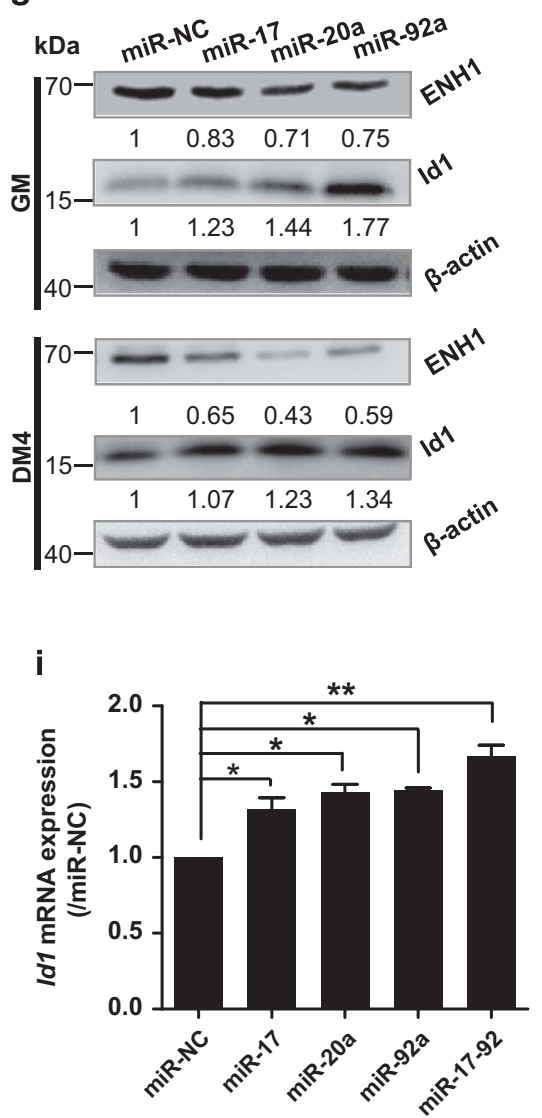

Figure 4 miR-17-92 and ENH1 modulate Id1 subcellular distribution. (a) Western blot analysis shows that the Id1 protein is downregulated during C2C12 myoblast myogenesis. (b) Nuclear and cytoplasmic Id1 proteins are downregulated in $\mathrm{C} 2 \mathrm{C} 12$ cells at DM4. Alpha-tubulin and TATA-binding protein (TBP) served as the loading controls. The data are presented as the means of the samples from three different cell samples. (c) Localization of exogenous Flag-ld1 in C2C12 myoblasts (cultured in GM) and in differentiating $\mathrm{C} 2 \mathrm{C} 12$ cells at DM4, as detected by Flag-ld1 immunostaining (red). Nuclei were stained blue with DAPI. Scale bar, $50 \mu \mathrm{m}$. (d) si-ENH1 increases the nuclear FlagId1 in $\mathrm{C} 2 \mathrm{C} 12$ myoblasts. C2C12 myoblasts were co-transfected with Flag-ld1 and si-ENH1 and transferred to DM for 4 days. The rest is as in c. (e) Western blot analysis of the whole-cell, nuclear and cytoplasmic Id1 proteins in C2C12 myoblasts transfected with si-ENH1 or NC. The rest is as in b. (f) Overexpression of miR-17, -20a or -92a induces the accumulation of Flag-ld1 in the nucleus. C2C12 myoblasts were co-transfected with Flag-ld1 and miRNA mimics and transferred to DM for 4 days. The rest is as in $\mathbf{c}$. (g) Overexpression of miR-17, -20a or -92a regulates the protein levels of ENH1 and Id1 in C2C12 cells that were transfected with miRNA mimics or NC at GM and at DM4. Beta-actin served as the loading control. The rest is as in b. (h) Western blot analysis of nuclear and cytoplasmic ld1 in the C2C12 myoblasts transfected with a mixture of miR-17, -20a and $-92 a$ mimics or NC. The rest is as in b. (i) Id1 mRNA is upregulated in C2C12 myoblasts stably expressed miRNA as indicated. The data are presented as the means \pm S.D. of the samples from three different cell samples. ${ }^{*} P<0.05$ and ${ }^{* *} P<0.01$

damaged area at 1 and 3 days post injury (Supplementary Figure S10). Therefore, miR-20a enhanced the proliferative activity of the muscle satellite cells, which is evidenced by the increases in Ki67 stained cells $(2.20$-fold, $P<0.01)$ and
PCNA protein levels (2.07-fold, $P<0.05)$ (Figures $5 d$ and e). As previously described, miR-20a was downregulated 3-10 days post injury (Figure 1d). We next investigated whether the overexpression of miR-20a would impact muscle 
a
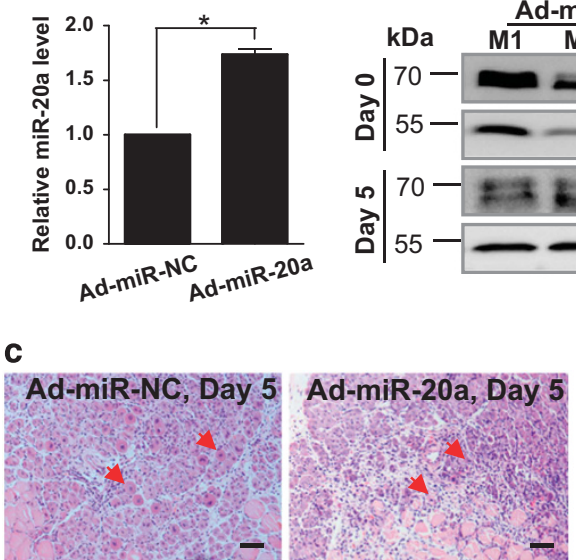

Ad-miR-NC, Day 10 Ad-miR-20a, Day 10

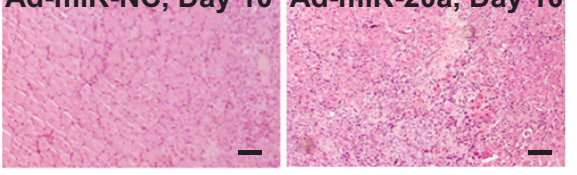

d

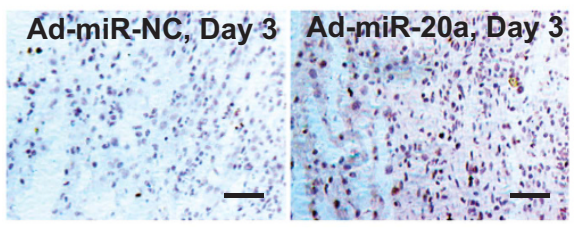

g
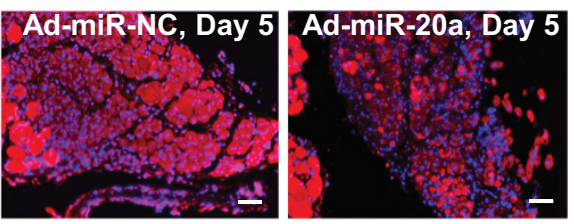

b

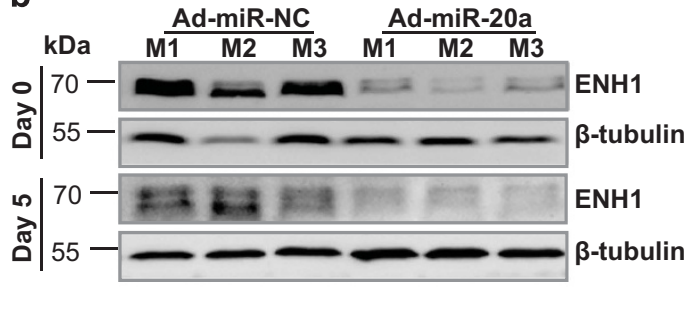

e
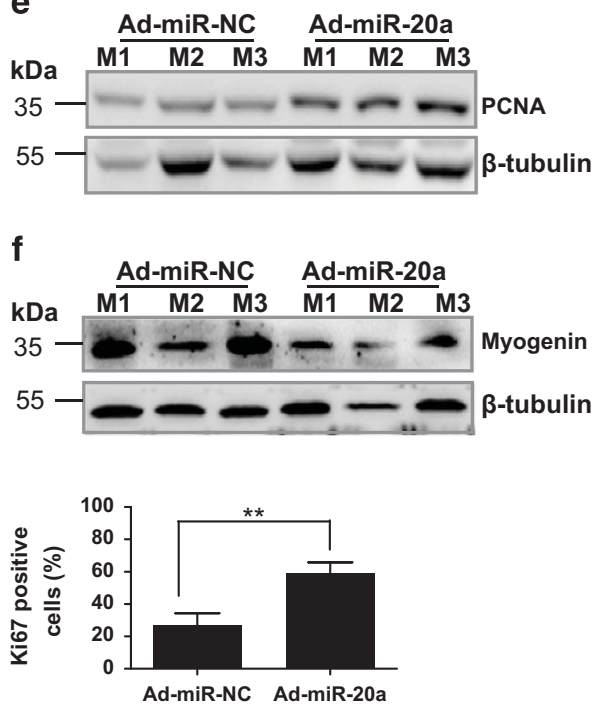

h
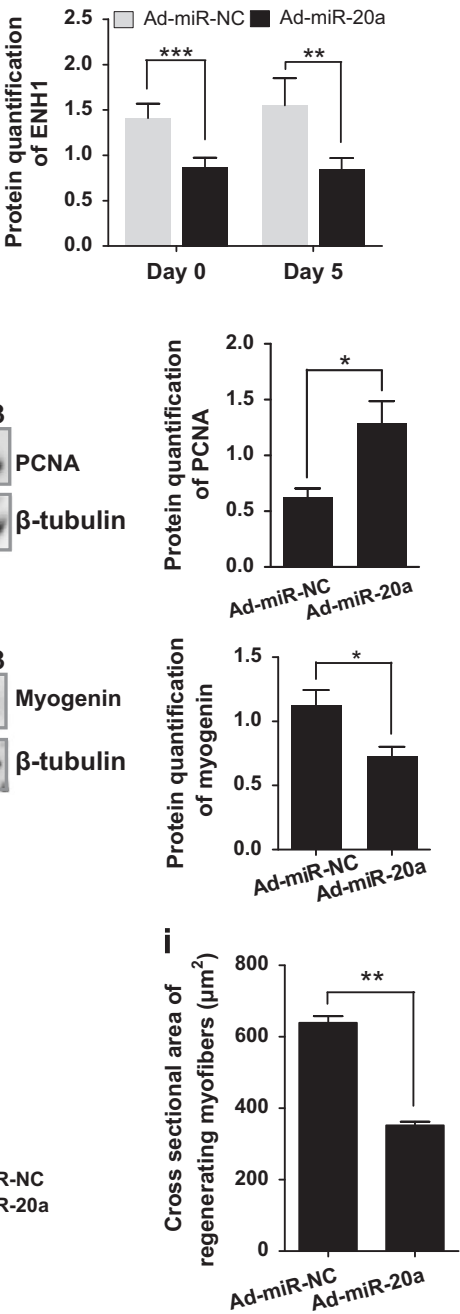

Figure 5 Ad-miR-20a delays skeletal muscle regeneration after the CTX injury. C57BL/6 mice were injected in their tibialis anterior muscles with CTX. Adenoviruses expressing miR-20a (Ad-miR-20a) or control (Ad-miR-NC) were injected into the tibialis anterior muscles 10 days prior to the CTX injection. The tibialis anterior muscles were collected on $0,1,3,5$ and 10 days post-CTX injury. (a) Quantitative PCR shows that the injection of Ad-miR-20a increases miR-20a in the mouse tibialis anterior muscles 10 days after the adenoviruses injection. qRT-PCR of miR-20a normalized to SNORNA234 is shown relative to control adenovirus-injected samples. The error bars depict the means \pm S.D. of the samples from six mice. ${ }^{*} P<0.05$. (b, left) Injection of Ad-miR-20a in the tibialis anterior muscles downregulates the ENH1 protein level as detected by western blot on post-CTX injury day 0 and 5 (top panel). M1, mouse 1; M2, mouse 2; M3, mouse 3. Beta-tubulin served as the loading control. (Right) The ENH1 proteins were quantified, and the data were expressed relative to the control adenovirus-injected (Ad-miR-NC) samples. The data are presented as the means + S.D. of the samples from five mice. ${ }^{* *} P<0.01$ and ${ }^{* * *} P<0.001$. (c) H\&E images of the tibialis anterior muscle cross-sections of mice injected with Ad-miR-NC or Ad-miR-20a on 5 and 10 days post-CTX injury. Arrows indicate the regenerating myofibers. Scale bar, $200 \mu \mathrm{m}$. (d, left) Immunostaining of Ki67 in the tibialis anterior muscle on day 3 after the CTX injury. Scale bar, $200 \mu \mathrm{m}$. (Right) Quantification of Ki67-positive cells from 10 random fields per sample. The error bars depict the means \pm S.D. of the samples from six mice. ${ }^{*} P<0.05$. (e, left) Western blot for PCNA in the tibialis anterior muscles on day 1 after the CTX injury. (Right) Quantification of PCNA protein levels. Ad-miR-20a increased the protein levels of PCNA in the tibialis anterior muscle. The results were normalized to beta-tubulin and expressed relative to the control. The error bars depict the means \pm S.D. of samples from five mice. ${ }^{*} P<0.05$. (f) AdmiR-20a downregulated the protein expression of myogenin in the tibialis anterior muscles on day 3 post-CTX injury. Western blot (left) and the quantification (right) of myogenin in the tibialis anterior muscles. The rest is as in $\mathbf{e}$. (g) Immunostaining of MHC in regenerating fibers on day 5 post-CTX injury. Scale bar, $200 \mu \mathrm{m}$. (h) Analysis of the cross-sectional areas of regenerating fibers on day 5 post injury using the Image $J$ software. Only myofibers containing centralized nuclei were measured. The error bars depict the means \pm S.D. of samples from five mice. (i) Average area of the cross-sections of regenerating fibers on day 5 post-CTX injury. Approximately eight random fields were captured, and $>1500$ fibers were measured per sample. The error bars depict the means \pm S.D. of samples from five mice. ${ }^{* \star} P<0.01$

differentiation as was observed in vitro. Under normal conditions, new myofibers containing central nuclei were formed to repair damaged fibers on day 5 post injury (Supplementary Figure S2). However, Ad-miR-20a downregulated the protein levels of myogenin $(0.65$-fold, $P<0.05)$ in the tibialis anterior muscle (Figure 5f) and delayed the growth of new myofibers (Figures $5 \mathrm{c}$ and $\mathrm{g}$ ), which is evidenced by the analysis of myosin-stained cross-sectional areas revealing a decrease in myofiber sizes in the Ad-miR-20a muscle (Figure 5h), with an average size of $351.7 \pm 10.6$ versus $638.8 \pm 19.2 \mu \mathrm{m}^{2}$ 
a

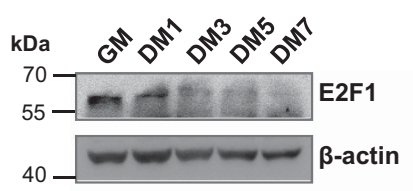

d
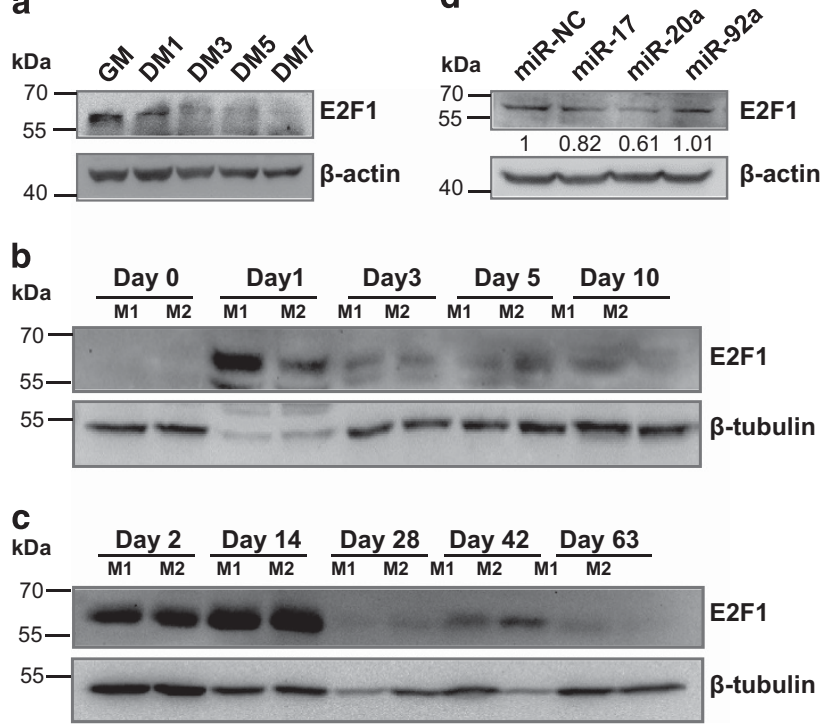

Figure 6 E2F1 increases the transcription of miR-17-92 in myoblasts. (a-c) E2F1 protein expression during $\mathrm{C} 2 \mathrm{C} 12$ myoblast myogenesis (a), mouse muscle regeneration (b) and postnatal growth (c). Beta-actin and beta-tubulin served as the loading controls. (d) Overexpression of miR-17, -20a or -92a reduces E2F1 protein expression in $\mathrm{C} 2 \mathrm{C} 12$ myoblasts transfected with the miR-17, -20a or -92a mimics. The data are presented as the means of the samples from three different cell samples. M1, mouse 1; M2, mouse 2. Beta-actin served as the loading control

(Ad-miRNA-20a versus control muscle, $P<0.01$; Figure $5 \mathrm{i}$ ). These results indicate that miR-20a represses muscle differentiation during muscle regeneration. At 10 days post injury, the majority of the damaged myofibers were repaired in the control muscle, whereas abundant mononucleated cells and regenerating myofibers persisted in the Ad-miR-20a muscle (Figure 5c), indicating that the abnormal high expression of miR-20a delays muscle regeneration. Taken together, and as found in vitro, the overexpression of miR-20a enhances muscle proliferation but delays muscle regeneration by inhibiting its target ENH1 in vivo.

miR-17-92 expression is positively regulated by E2F1 in skeletal muscle. E2F1 regulates miR-17-92 expression in various cell systems. ${ }^{30,31}$ We found that the downregulation of miR-17-92 was accompanied by a decrease in E2F1 protein during $\mathrm{C} 2 \mathrm{C} 12$ myoblast myogenesis (Figure 6a). Moreover, E2F1 was downregulated during muscle regeneration and postnatal development in mice (Figures $6 \mathrm{~b}$ and $\mathrm{c}$ ). We carried out a miR-17-92 promoter luciferase assay. The overexpression of E2F1 induced an increase in the luciferase activity (8.06-fold, $P<0.001)$, whereas the elimination of E2F1-binding sites from the miR-17-92 promoter abolished this activation (Supplementary Figure S11). In contrast, the mimics of miR-17 and -20a, but not of miR-92a, reduced the E2F1 protein level in $\mathrm{C} 2 \mathrm{C} 12$ myoblasts (Figure 6d). These results show that E2F1 enhances the transcription of miR-17-92, whereas miR-1792 induces a negative feedback loop and inhibits E2F1 in skeletal muscle cells.

\section{Discussion}

In this study, we analyzed the expression pattern of 720 miRNAs in mouse C2C12 myoblasts before and during differentiation and identified miR-17-92, a cluster of six miRNAs that was significantly downregulated, as an inhibitory myogenic miRNA. We demonstrated the pro-proliferative and anti-differentiation effects of miR-17-92 in vitro and in vivo and elucidated the E2F1/miR-17-92/ENH1/ld1 regulatory axis in muscle myogenesis, regeneration and development (Figure 7).

Of the 55 miRNAs differentially regulated in response to differentiation (Figure 1a), 16 miRNAs have previously been shown to play functional roles in myogenesis, including miR-1, $-133,-24,-26 a,-27,-29,-206$ and $-486,{ }^{9,32-34}$ indicating that this novel method is successful in profiling miRNA expression patterns in a high-throughput manner. Therefore, this study offers a new strategy to efficiently identify myogenesis-associated miRNAs. Some of the additional miRNAs unstudied herein will be investigated in future studies.

Previous studies reveal a critical requirement for miR-17-92 at the early stage of development. A similar expression pattern has also been found during the normal development of lung and heart. ${ }^{17,25}$ miR-17-92 knockout causes lung hypoplasia, cardiac defects and a reduction of pre-B cells, which subsequently results in early-postnatal lethality. ${ }^{17}$ Moreover, miR-17-92-deleted embryos exhibit multiple skeletal abnormalities. ${ }^{17}$ Given the anti-differentiation role reported of other downregulated miRNAs during myogenic differentiation, including miR-155, -669a, -669q, -487b, -3963 and $-6412,{ }^{10,35,36}$ the reduction in these miRNAs at the initial step of myogenesis can be considered a miRNA signature of antidifferentiation effectors. In contrast, the differentiation-induced miRNAs, such as miR-1, -27 -26a, -29, -206 and -378, consistently induce the opposite effect on myogenesis. ${ }^{11,37-41}$ Surprisingly, some upregulated miRNAs, such as miR-133, have repressive abilities on myogenic differentiation. ${ }^{37}$ Regardless, the principal effects of miRNAs on myogenesis are the modulation of cell proliferation and differentiation.

Muscle differentiation and regeneration are highly dependent on the pro-proliferative capacity of a pool of muscle cells. In vitro studies demonstrated that the expression of MyoD and myogenin was upregulated in a density-dependent manner in C2C12 myoblasts. ${ }^{42}$ At a low-cell density, Myf5 inhibited the induction of myogenin in the human U20S cells. ${ }^{43}$ Moreover, the number of regenerating myofibers and the expression of MyoD and myogenin are decreased when the proliferative ability of the satellite cells is inhibited, for example, by angiotensin II. ${ }^{44}$ These studies suggest that sufficient numbers of progenitor cells are required for myogenesis and muscle regeneration. Given the high expression of miR-17-92 at the early stage of myogenesis and regeneration (Figures 1c and $\mathrm{d}$ ), one might have predicted that its high expression would favor the accumulation of muscle progenitor cells. Conversely, our skeletal regeneration experiments reveal that the abnormal high expression of miR-20a caused a hyperproliferative phenotype and impeded muscle differentiation and myofiber maturation. Similarly, the transgenic overexpression of the miR-17-92 cluster in mice leads to the hyperproliferation of lung epithelium, the inhibition of differentiation 


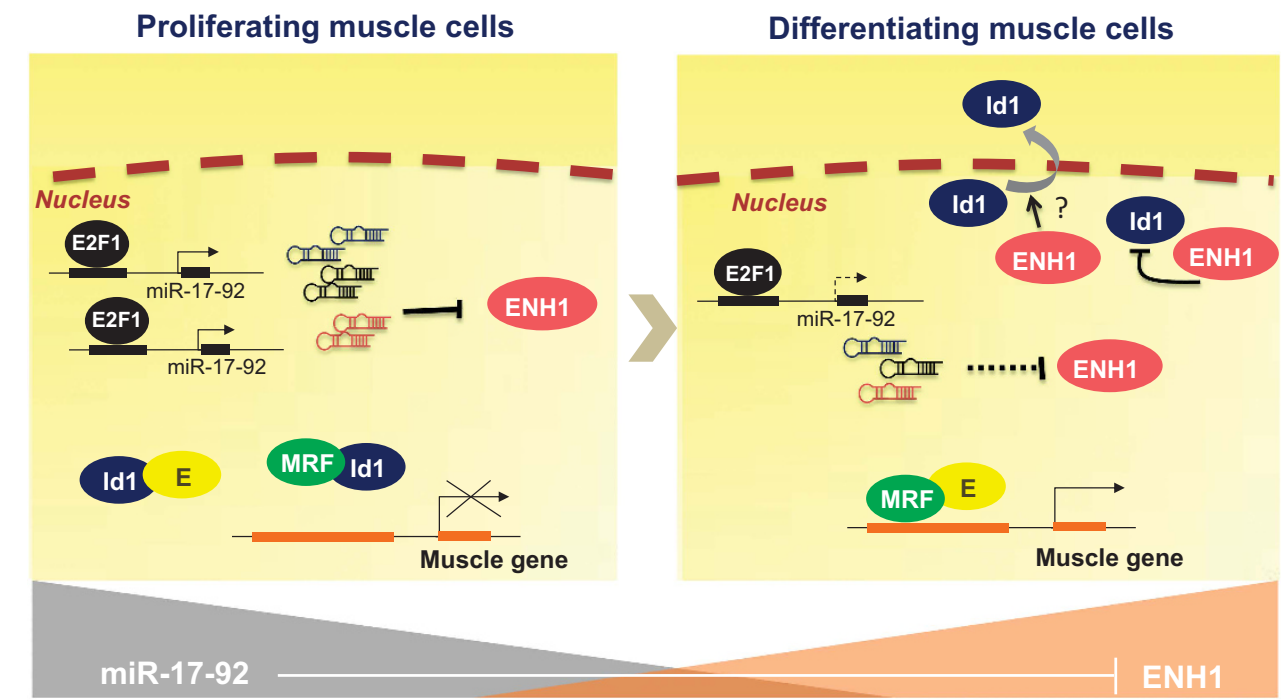

Figure 7 A model of the E2F1/miR-17-92/ENH1/ld1 regulatory axis in myogenesis. In the proliferating myoblasts, E2F1 upregulates the transcription of miR-17-92. miR-17, -20a and -92a, sequester Id1 in the nucleus by targeting its cytoplasmic sequestration factor, ENH1. Id1 binds to myogenic regulatory factor (MRF) and E protein, which prevents the heterodimerization of MRF and E protein and subsequently inhibits myogenic differentiation. Upon the stimulation of differentiation, the low expression of miR-17-92 results in a high level of ENH1, which reduces the abundance of Id1 protein in the nucleus, possibly by sequestrating the Id1 into the cytoplasm. The complex of MRF and E protein binds to the promoters of target muscle genes, thereby driving their expressions and inducing myogenic differentiation

and subsequently an abnormal lethal phenotype characterized by the absence of air in the lungs. ${ }^{25}$

ENH1 is a heart/skeletal muscle-specific PDZ-LIM protein and is implicated in heart and skeletal muscle development. ${ }^{45-48}$ Yoshida and co-workers ${ }^{48}$ have shown that this protein promotes the mRNA levels of myoD and myogenin in $\mathrm{C} 2 \mathrm{C} 12$ cells. ENH1 has also been shown to have antiproliferative effects on human neuroectodermal cell lines. ${ }^{28}$ Here, we confirmed that ENH1 has anti-proliferative and prodifferentiation effects in myoblasts. The sequestration function of ENH1 is attributed to specific protein domains, with the PDZ domain of ENH1 binding to cytoskeletal proteins (such as actin and alpha-actinin) allowing for the organization of protein complexes at the cytoskeleton, ${ }^{45}$ and the LIM domain interacting with diverse partners. ${ }^{28,49}$ Previous studies have shown that alternative PDZ-LIM proteins could contribute to the regulation and subcellular compartmentalization of nuclear proteins. ${ }^{46}$ As mentioned above, ENH1 has been linked to the cytoplasmic localization of Id2 in neuronal cells. ${ }^{28}$ In our study, we highlighted the negative effect of ENH1 on the nuclear abundance of Id1 (Figure 7). It should be noted that this study investigated only Id1. In fact, Id1 and Id 3 can both bind to $\mathrm{ENH} 1,{ }^{28}$ and the regulation of these Id proteins by their subcellular localization has been demonstrated to modulate cell proliferation and differentiation. ${ }^{50-52}$ Therefore, the effect of ENH1 on the localization of Id1, Id2 and Id 3 during myogenesis remains to be determined (Figure 7).

$\mathrm{E} 2 \mathrm{~F} 1$, along with E2F2 and E2F3, bind to the promoter of miR-17-92 to activate its transcription, and miR-17 and $-20 \mathrm{a}$ simultaneously repress the translation of E2F1-3 via 3'UTRbinding sites, ${ }^{30,31}$ establishing an auto-regulatory feedback loop between E2F1 and miR-17-92. Here we confirmed the positive regulation of E2F1 on miR-17-92 transcription (Figures $6 \mathrm{a}-\mathrm{C}$ and Supplementary Figure S10), highlighting that miR-17-92 expression is induced by E2F1 during muscle differentiation, regeneration and postnatal development. However, E2F1 protein can be inhibited by miR-17 and $-20 a$, but not by miR-92a (Figure 6d), further validating the E2F1miR-17/20a feedback loop in skeletal muscle cells.

In conclusion, we demonstrated the miRNA expression patterns in response to differentiation and identified the members of the miR-17-92 cluster as myogenesis-associated miRNAs. We then dissected the function of miR-17-92 in controlling muscle proliferation, differentiation and regeneration and discovered the E2F1/miR-17-92/ENH1/ld1 regulatory axis in myogenesis.

\section{Materials and Methods}

Cell culture. The HEK 293A, HEK 293T and C2C12 cells were purchased from American Type Culture Collection (ATCC, Manassas, VA, USA). The C2C12 cells were cultured in $\mathrm{GM}$ and transferred to DM for myogenesis. In brief, the $\mathrm{C} 2 \mathrm{C} 12$ cells were maintained in DMEM (Corning Cellgro, Manassas, VA, USA) supplemented with $10 \%$ fetal bovine serum (FBS, Biowest, Nuaillè, France) in a humidified incubator with $5 \% \mathrm{CO}_{2}$ at $37^{\circ} \mathrm{C}$. To induce myogenic differentiation, the culture medium was switched to DMEM with $2 \%$ heat-inactivated horse serum (Gibco, Grand Island, NY, USA). The 293A and 293T cells were cultured in DMEM with 10\% FBS in a humidified incubator with $5 \% \mathrm{CO}_{2}$ at $37^{\circ} \mathrm{C}$.

Animals and muscle collection. The C57BL/6 and BALB/C male mice were purchased from the Guangdong Medical Laboratory Animal Center (Guangzhou, China). The mice were used at 2-10 weeks of age and were agematched for each independent experiment. The castrated male hybrid pigs (Landrace $\times$ Large White $\times$ Duroc) were used at 35-161 days of age and maintained at the South China Agricultural University. The use of animals was in accordance with the recommendations of the Guide for the Care and Use of Laboratory Animals of China. All the protocols were approved by the Animal Ethical and Welfare Committee of Shenzhen University (Approval No. AEWC-2014-001004). The pigs were housed in a controlled environment and were provided free access to food and water. The experimental diet was designed according to the NRC2012 (Nutrient requirements of swine, 2012). The porcine longissimus dorsi muscles were 
collected at postnatal days $35,63,98$ and 161 . The hind leg muscles of the BALB/C mice were collected at postnatal days $14,28,42$ and 63 . There are at least six pigs or mice in each group. The muscle samples were immediately frozen in liquid nitrogen and stored at $-80^{\circ} \mathrm{C}$.

Mouse muscle regeneration model and adenovirus injection. Muscle degeneration and regeneration in mice were induced by injecting cardiotoxin (CTX). Six week-old C57BL/6 mice were injected in their tibialis anterior muscles with $100 \mu \mathrm{l}$ of $10 \mu \mathrm{M} \mathrm{CTX}$. Adenoviruses expressing miR-20a or negative control $\left(10^{10}\right.$ to $10^{11}$ p.f.u./ml) were injected into the tibialis anterior muscles 10 days prior to the CTX injection. Two tibialis anterior muscles from each mouse were collected at $0,1,3,5$ and 10 days post-CTX injury. One sample was fixed in $4 \%$ paraformaldehyde for histology and immunohistochemistry. The other sample was frozen in liquid nitrogen and stored at $-80^{\circ} \mathrm{C}$ for RNA and protein extraction.

RNA extraction and qRT-PCR. Total RNA was extracted with the RNAiso Reagent (TaKaRa, Dalian, China) according to the manufacturer's instructions. The isolated RNA was quantified using the NanoDrop 2000c Spectrophotometer (Thermo Fisher Scientific, Wilmington, DE, USA). miRNA quantification was performed by qRT-PCR based on the S-Poly(T) Plus method (Geneups, Shenzhen, Guangdong, China). ${ }^{15}$ SNORNA44, SNORNA202 and SNORNA234 were used as the human, porcine and mouse miRNA endogenous controls, respectively. The SYBR Green method was used for mRNA quantitative determination using oligo(dT) plus random primers to initiate CDNA synthesis. RPL-14 was selected as a normalization control. The PCR reactions were carried out in the StepOnePlus Real-Time PCR System (Applied Biosystems, Grand Island, NY, USA). The primers used for reverse transcription and qPCR can be found in Supplementary Table S1.

\section{Plasmids}

ENH1 3'UTR luciferase reporter assay. To construct the ENH1 3'UTR luciferase reporter plasmid, the ENH1 $3^{\prime}$ UTR was amplified from mouse genomic DNA using the primer listed in Supplementary Table S2. The purified PCR products were then cloned into the downstream region of the Firefly luciferase reporter gene at the $E c o R I / X b a l$ restriction sites of the miRGlo vector (Promega). The corresponding mutant constructs with 6-7 mutated residues in the predicted binding site were generated by site-directed mutagenesis using the primer sets listed in Supplementary Table S2.

Myogenin promoter luciferase assay. To construct the myogenin promoter-driven luciferase reporter vector, a fragment $(1.1 \mathrm{~kb})$ of the mouse myogenin promoter was subcloned into the basic vector pGL4 (Promega) via the Xhol/Mlul sites. The myogenin promoter was amplified from mouse genomic DNA by using the primers listed in Supplementary Table S2.

miR-17-92 promoter luciferase assay. To construct the miR-17-92 promoter-driven luciferase reporter vector, a fragment (250 bp) of miR-17-92 promoter that contains two E2Fs-binding sites was amplified from human genomic DNA using the primers listed in Supplementary Table S2, then was subcloned into the vector pGL4 via the $\mathrm{Kpnl} / \mathrm{Mlul}$ sites. The mutant construct with mutated residues in the two E2Fs-binding sites was generated with the primers listed in Supplementary Table S2.

miRNA overexpression and inhibition. The lentiviral vectors expressing miR-17, $-20 \mathrm{a},-92 \mathrm{a}$ or $-17-92$ were constructed by inserting the primary miRNAs into the pLVX-Puro vector (Clontech, Mountain View, CA, USA). To monitor for the transduction efficiency, the open reading frame of EGFP containing a stop codon was inserted between the CMV promoter and the primary miRNAs. The pLVX-miRNC containing the EGFP fragment without any miRNA sequences was used as a negative control vector. The adenoviral vectors (Ad-miR-20a and Ad-miR-NC) were constructed with the BLOCK-iT Adenoviral RNAi Expression System (Invitrogen, Carlsbad, CA, USA). The lentiviral-based miRNA inhibitors dTuD-miR-17, -20a or -92a were constructed based on the Tough Decoy (TuD) design, ${ }^{53}$ using the two-step PCR method. ${ }^{54}$

Lentivirus packaging and transduction. The lentiviral particles were packaged in HEK $293 \mathrm{~T}$ cells by the transfection of the following three individual plasmids at a ratio of 2:1:5-(i) psPAX2 encoding HIV Gag-Pol (Addgene plasmid 12260; Addgene), (ii) pVSVg encoding the VSV-G glycoprotein (Addgene plasmid 8454; Addgene), and (iii) a lentiviral vector. Briefly, the HEK 293 T cells were seeded in $10-\mathrm{cm}$ culture dishes at a density of $4 \times 10^{6}$ cells per dish. After $24 \mathrm{~h}$ of incubation, the cells were transfected with lentiviral vectors $(12.5 \mu \mathrm{g})$ and the packing plasmids $(7.5 \mu \mathrm{g})$ using the PEI reagents. The cell-free supernatants were then harvested 48 and $72 \mathrm{~h}$ after transfection and used for subsequent cell infection in the presence of $8 \mu \mathrm{g} / \mathrm{ml}$ of polybrene. The infected cells were then selected by supplementing the culture medium with $1-2 \mu \mathrm{g} / \mathrm{ml}$ of puromycin $48 \mathrm{~h}$ after infection. The efficiency of the overexpression/inhibition of miRNAs was confirmed by qRTPCR analysis.

Transfection and luciferase reporter assays. The synthetic miRNA mimics and siRNAs were purchased from RiboBio (Guangzhou, Guangdong, China). The miRNA mimics or siRNAs were transfected into HEK 293A or C2C12 cells using the K2 transfection system (Biontex Laboratories $\mathrm{GmbH}$, München, Germany). The transfection of plasmids into $\mathrm{C} 2 \mathrm{C} 12$ cells was performed with the $\mathrm{PEI}$ transfection reagent following the manufacturer's instructions. For the myogenin promoter luciferase reporter assay, $\mathrm{C} 2 \mathrm{C} 12$ cells were co-transfected with plasmids overexpressing miRNA, myogenin promoter luciferase reporter plasmids and internal control of in-house modified SV40 promoter-driven Renilla luciferase vector (pSV40-R.Luc). For ENH1 3'UTR luciferase assays, C2C12 cells were transfected with miRNA mimics and ENH1 $3^{\prime}$ UTR or mutated ENH1 $3^{\prime}$ UTR reporter plasmids. At $48 \mathrm{~h}$ post transfection, the measurement of luciferase activity was performed with a luminometer Lumat3 LB9508 (Berthold Technologies, Bad Wildbad, Germany) using a dual-luciferase reporter assay system (Promega) following the manufacturer's instructions. The relative luciferase activities were calculated by comparing the Firefly/Renilla luciferase ratio.

Cell proliferation analysis. The EdU incorporation assay was performed using the EdU assay kit (Ribobio) according to the manufacturer's instructions. Briefly, 24 or $48 \mathrm{~h}$ after transfection, the $\mathrm{C} 2 \mathrm{C} 12$ cells were cultured in GM containing EdU $(50 \mu \mathrm{M})$ for $1.5 \mathrm{~h}$. The EdU labeling was conducted for $1 \mathrm{~h}$ for the $\mathrm{C} 2 \mathrm{C} 12$ cells stably expressing miRNA or dTuD-miRNA. The $\mathrm{C} 2 \mathrm{C} 12$ cells were further analyzed by calculating their EdU incorporation.

Immunoblotting, cell immunostaining and immunohistochemistry

Western blot. The total proteins of the $\mathrm{C} 2 \mathrm{C} 12$ cells or muscle samples were extracted with RIPA lysis buffer (50 mM Tris- $\mathrm{HCl}, \mathrm{pH} 7.5,150 \mathrm{mM} \mathrm{NaCl}, 1 \% \mathrm{NP}-40$, $0.25 \%$ sodium deoxycholate and $1 \mathrm{mM}$ EDTA) supplemented with protease inhibitor cocktail (Roche, Mannheim, Germany) and quantified using the bicinchoninic acid (BCA) protein assay kit (Thermo Scientific, Beijing, China). The separation and preparation of cytoplasmic and nuclear protein from $\mathrm{C} 2 \mathrm{C} 12$ were performed with NE-PER nuclear and cytoplasmic extraction reagents kit (Thermo Scientific) The proteins were electrophoresed with SDS-PAGE and transferred to PVDF membranes. After blocking with $5 \%$ skim milk in TBST $(20 \mathrm{mM}$ Tris. $\mathrm{HCl}, \mathrm{pH} 7.6$, $150 \mathrm{mM} \mathrm{NaCl}$, and $0.1 \%$ Tween 20), the blots were incubated with primary antibodies and then with HRP-conjugated secondary antibodies. The protein bands were visualized using the SuperSignal chemiluminescent detection module (Pierce Biotechnology, Rockford, IL, USA), and images were captured with the Tanon-5200 imaging system (Tanon, Shanghai, China). The following primary antibodies were used: $\beta$-actin (1: 10 000; Proteintech, Wuhan, Hubei, China), $\beta$-tubulin (1:5,000; Proteintech), E2F1 (1: 600; Santa Cruz Biotechnology, Santa Cruz, CA, USA), ENH1 (1:300; Proteintech), Id1 (1: 100; CalBioreagents, San Mateo, CA, USA), myogenin (1:5,000; Abcam, Cambridge, MA, USA) and PCNA (1:2,000; Proteintech). The HRP-conjugated secondary antibodies (1:10 000; Bio-Rad, Hercules, CA, USA) were also used.

Immunostaining: The immunostaining of cells was carried out with slight modifications of the previously reported protocols. ${ }^{38}$ The $\mathrm{C} 2 \mathrm{C} 12$ cells were grown on sterile glass coverslip in 24-well plates, fixed with $4 \%$ formaldehyde in PBS for $20 \mathrm{~min}$ at room temperature and permeabilized with $0.5 \%$ Triton X-100 in ice-cold PBS for $10 \mathrm{~min}$. The pretreated cells were blocked with $1 \%$ bovine serum albumin for $15 \mathrm{~min}$ and incubated with primary antibodies for $3 \mathrm{~h}$ at room temperature or overnight at $4{ }^{\circ} \mathrm{C}$ at the following dilutions: anti-Myosin heavy chain $(1: 100$; Abcam) and anti-Flag (1:25; Proteintech). The indirect immunofluorescence was detected after incubation with fluorescein isothiocyanate-conjugated anti-mouse/ rabbit IgG (1:500; Abcam). The cell nuclei were stained with 4',6-Diamidino-2phenylindole dihydrochloride (DAPI) for 5 min. After several washes with PBS, the cells were subjected to a fluorescence microscope (Carl Zeiss, Oberkochen, Germany).

Immunohistochemical staining: Immunohistochemical staining of skeletal muscle tissue sections was performed as previously described. ${ }^{55,56}$ The muscle tissue sections (paraffin-embedded) were deparaffinized and treated with the heat- 
induced epitope retrieval (HIER) antigen retrieval EDTA-Tris buffer (pH 9.0). After retrieval, the sections were then incubated with the primary antibodies (anti-BrdU (1:50; BD Pharmingen, San Diego, CA, USA) and rabbit anti-Ki67 (1:100; Abcam)). The $3^{\prime}, 3$ diaminobenzidine (DAB) substrate was used as a chromogenic substrate for the detection of HRP. Images were captured with a fluorescence microscope.

Histology. The paraffin-embedded skeletal muscle tissue samples were cut into $5-\mu \mathrm{m}$-thick sections, mounted and stained with H\&E. Images were captured using a fluorescence microscope. The determination of the area of regenerating fibers was performed with at least 30 fields from 5 sections of the tibialis anterior muscle in 5 mice for each group. The analysis of the fiber area was performed with the ImageJ software (National Institute of Mental Health, Bethesda, MD, USA).

Statistical analysis. All results are expressed as the mean of at least three triplicates for each treatment. Pairwise comparisons were performed using a twotailed Student's $t$-test with STATGRAPHICS (Centurion XVIII) software (StatPoint Technologies, Warrenton, VA, USA). A $P$-value of $<0.05$ was considered statistically significant.

\section{Conflict of Interest}

The authors declare no conflict of interest.

Acknowledgements. We would like to thank Yuefang Jiang of ShenZhen University for providing technical assistance with the high-throughput miRNA screen and Dingyuan Feng of South China Agricultural University for providing us the porcine skeletal muscles. We thank Prof. Bin Tang in South University of Science and Technology for technical supports. This work was supported by the National Basic Research Program of China (973 Program) (2012CB124701 to DG); the National Natural Science Foundation of China (81170047, 81370151 and 81570046 to DG; and 31571199 to KK); the Shenzhen Municipal Basic Research Program (JCYJ20150729104027220 to DG); the Shenzhen Overseas High-Level Talents Innovation Program (YFZZ20111009 to DG); and the Shenzhen High-tech Development Project (CXZZ20140828163951592 to DG).

1. Moss FP, Leblond CP. Satellite cells as the source of nuclei in muscles of growing rats. Anat Rec 1971; 170: 421-435.

2. White RB, Bierinx AS, Gnocchi VF, Zammit PS. Dynamics of muscle fibre growth during postnatal mouse development. BMC Dev Biol 2010; 10: 10-21.

3. Le Grand F, Rudnicki MA. Skeletal muscle satellite cells and adult myogenesis. Curr Opin Cell Biol 2007; 19: 628-633.

4. Sabourin LA, Rudnicki MA. The molecular regulation of myogenesis. Clin Genet 2000; 57 : $16-25$.

5. Olson EN. Interplay between proliferation and differentiation within the myogenic lineage. Dev Biol 1992; 154: 261-272.

6. Langlands K, Yin X, Anand G, Prochownik EV. Differential interactions of Id proteins with basic-helix-loop-helix transcription factors. J Biol Chem 1997; 272: 19785-19793.

7. Gundersen K, Rabben I, Klocke BJ, Merlie JP. Overexpression of myogenin in muscles of transgenic mice: interaction with Id-1, negative crossregulation of myogenic factors, and induction of extrasynaptic acetylcholine receptor expression. Mol Cell Biol 1995; 15 : 7127-7134.

8. Sato T, Yamamoto T, Sehara-Fujisawa A. miR-195/497 induce postnatal quiescence of skeletal muscle stem cells. Nat Commun 2014; 5: 4597.

9. Luo W, Nie $Q$, Zhang X. MicroRNAs involved in skeletal muscle differentiation. J Genet Genomics 2013; 40: 107-116.

10. Katase N, Terada K, Suzuki T, Nishimatsu S, Nohno T. miR-487b, miR-3963 and miR-6412 delay myogenic differentiation in mouse myoblast-derived C2C12 cells. BMC Cell Biol 2015; 16: 13 .

11. Dey BK, Gagan J, Yan Z, Dutta A. miR-26a is required for skeletal muscle differentiation and regeneration in mice. Genes Dev 26: 2180-2191.

12. Liu N, Williams AH, Maxeiner JM, Bezprozvannaya S, Shelton JM, Richardson JA et al. microRNA-206 promotes skeletal muscle regeneration and delays progression of Duchenne muscular dystrophy in mice. J Clin Invest 2012; 122: 2054-2065.

13. van Rooij E, Quiat D, Johnson BA, Sutherland LB, Qi X, Richardson JA et al. A family of microRNAs encoded by myosin genes governs myosin expression and muscle performance. Dev Cell 2009; 17: 662-673.

14. Kang K, Zhang X, Liu H, Wang Z, Zhong J, Huang Z et al. A novel real-time PCR assay of microRNAs using S-Poly $(T)$, a specific oligo(dT) reverse transcription primer with excellent sensitivity and specificity. PLOS One 2012; 7: e48536.
15. Niu Y, Zhang L, Qiu H, Wu Y, Wang Z, Zai Y et al. An improved method for detecting circulating microRNAs with S-Poly(T) Plus real-time PCR. Sci Rep 2015; 5: 15100.

16. Hayashita $Y$, Osada $H$, Tatematsu $Y$, Yamada $H$, Yanagisawa $K$, Tomida $S$ et al. A polycistronic microRNA cluster, miR-17-92, is overexpressed in human lung cancers and enhances cell proliferation. Cancer Res 2005; 65: 9628-9632.

17. Ventura A, Young AG, Winslow MM, Lintault L, Meissner A, Erkeland SJ et al. Targeted deletion reveals essential and overlapping functions of the miR-17 through 92 family of miRNA clusters. Cell 2008; 132: 875-886.

18. Xiao C, Srinivasan L, Calado DP, Patterson HC, Zhang B, Wang J et al. Lymphoproliferative disease and autoimmunity in mice with increased miR-17-92 expression in lymphocytes. Nat Immunol 2008; 9: 405-414.

19. Matsubara H, Takeuchi T, Nishikawa E, Yanagisawa K, Hayashita Y, Ebi H et al. Apoptosis induction by antisense oligonucleotides against miR-17-5p and miR-20a in lung cancers overexpressing miR-17-92. Oncogene 2007; 26: 6099-6105.

20. Charge SB, Rudnicki MA. Cellular and molecular regulation of muscle regeneration. Physiol Rev 2004; 84: 209-238.

21. Yaffe $D$, Saxel $O$. Serial passaging and differentiation of myogenic cells isolated from dystrophic mouse muscle. Nature 1977; 270: 725-727.

22. Andres V, Walsh K. Myogenin expression, cell cycle withdrawal, and phenotypic differentiation are temporally separable events that precede cell fusion upon myogenesis. J Cell Biol 1996; 132: 657-666.

23. Kirby TJ, Chaillou T, McCarthy JJ. The role of microRNAs in skeletal muscle health and disease. Front Biosci (Landmark Ed) 2015; 20: 37-77.

24. Dmitriev P, Barat A, Polesskaya A, O'Connell MJ, Robert T, Dessen $P$ et al. Simultaneous miRNA and mRNA transcriptome profiling of human myoblasts reveals a novel set of myogenic differentiation-associated miRNAs and their target genes. BMC Genomics 2013; 14: 265.

25. Lu Y, Thomson JM, Wong HY, Hammond SM, Hogan BL. Transgenic over-expression of the microRNA miR-17-92 cluster promotes proliferation and inhibits differentiation of lung epithelial progenitor cells. Dev Biol 2007; 310: 442-453.

26. Alway SE, Degens H, Lowe DA, Krishnamurthy G. Increased myogenic repressor Id mRNA and protein levels in hindlimb muscles of aged rats. Am J Physiol Regul Integr Comp Physiol 2002; 282: R411-R422.

27. Benezra R, Davis RL, Lockshon D, Turner DL, Weintraub H. The protein Id: a negative regulator of helix-loop-helix DNA binding proteins. Cell 1990; 61: 49-59.

28. Lasorella A, lavarone $A$. The protein ENH is a cytoplasmic sequestration factor for Id2 in normal and tumor cells from the nervous system. Proc Natl Acad Sci USA 2006; 103: 4976-4981.

29. Sun L, Trausch-Azar JS, Ciechanover A, Schwartz AL. Ubiquitin-proteasome-mediated degradation, intracellular localization, and protein synthesis of MyoD and Id 1 during muscle differentiation. J Biol Chem 2005; 280: 26448-26456.

30. Sylvestre Y, De Guire V, Querido E, Mukhopadhyay UK, Bourdeau V, Major F et al. An E2F/ miR-20a autoregulatory feedback loop. J Biol Chem 2007; 282: 2135-2143.31.

31. Woods K, Thomson JM, Hammond SM. Direct regulation of an oncogenic micro-RNA cluster by E2F transcription factors. J Biol Chem 2007; 282: 2130-2134.

32. Sun $Q$, Zhang $Y$, Yang $G$, Chen $X$, Zhang $Y$, Cao $G$ et al. Transforming growth factor-betaregulated miR-24 promotes skeletal muscle differentiation. Nucleic Acids Res 2008; 36: 2690-2699.

33. Lee SW, Yang J, Kim SY, Jeong HK, Lee J, Kim WJ et al. MicroRNA-26a induced by hypoxia targets HDAC6 in myogenic differentiation of embryonic stem cells. Nucleic Acids Res 2015; 43: 2057-2073

34. Amirouche A, Tadesse H, Miura P, Belanger G, Lunde JA, Cote J et al. Converging pathways involving microRNA-206 and the RNA-binding protein KSRP control post-transcriptionally utrophin A expression in skeletal muscle. Nucleic Acids Res 2014; 42: 3982-3997.

35. Seok HY, Tatsuguchi M, Callis TE, He A, Pu WT, Wang DZ. miR-155 inhibits expression of the MEF2A protein to repress skeletal muscle differentiation. J Biol Chem 2011; 286: 35339-35346.

36. Crippa S, Cassano M, Messina G, Galli D, Galvez BG, Curk T et al. miR669a and miR669q prevent skeletal muscle differentiation in postnatal cardiac progenitors. J Cell Biol 2011; 193: 1197-1212.

37. Chen JF, Mandel EM, Thomson JM, Wu Q, Callis TE, Hammond SM et al. The role of microRNA-1 and microRNA-133 in skeletal muscle proliferation and differentiation. Nat Genet 2006; 38: 228-233.

38. Winbanks CE, Wang B, Beyer C, Koh P, White L, Kantharidis $P$ et al. TGF-beta regulates miR-206 and miR-29 to control myogenic differentiation through regulation of HDAC4. J Biol Chem 2011; 286: 13805-13814.

39. Wei W, He HB, Zhang WY, Zhang HX, Bai JB, Liu HZ et al. miR-29 targets Akt3 to reduce proliferation and facilitate differentiation of myoblasts in skeletal muscle development. Cell Death Dis 2013; 4: e668.

40. Gagan J, Dey BK, Layer R, Yan Z, Dutta A. MicroRNA-378 targets the myogenic repressor MyoR during myoblast differentiation. J Biol Chem 2011; 286: 19431-19438.

41. Crist CG, Montarras D, Pallafacchina G, Rocancourt D, Cumano A, Conway SJ et al. Muscle stem cell behavior is modified by microRNA-27 regulation of Pax3 expression. Proc Natl Acad Sci USA 2009; 106: 13383-13387.

42. Tanaka K, Sato K, Yoshida T, Fukuda T, Hanamura K, Kojima N et al. Evidence for cell density affecting $\mathrm{C} 2 \mathrm{C} 12$ myogenesis: possible regulation of myogenesis by cell-cell communication. Muscle Nerve 2011; 44: 968-977. 
43. Lindon C, Albagli O, Pinset C, Montarras D. Cell density-dependent induction of endogenous myogenin (myf4) gene expression by Myf5. Dev Biol 2001; 240: 574-584.

44. Yoshida T, Galvez S, Tiwari S, Rezk BM, Semprun-Prieto L, Higashi Y et al. Angiotensin II inhibits satellite cell proliferation and prevents skeletal muscle regeneration. $J$ Biol Chem 2013; 288: 23823-23832.

45. Nakagawa N, Hoshijima M, Oyasu M, Saito N, Tanizawa K, Kuroda S. ENH, containing PDZ and LIM domains, heart/skeletal muscle-specific protein, associates with cytoskeletal proteins through the PDZ domain. Biochem Biophys Res Commun 2000; 272: 505-512.

46. Krcmery J, Camarata T, Kulisz A, Simon HG. Nucleocytoplasmic functions of the PDZ-LIM protein family: new insights into organ development. Bioessays 2010; 32: 100-108.

47. Niederlander N, Fayein NA, Auffray C, Pomies P. Characterization of a new human isoform of the enigma homolog family specifically expressed in skeletal muscle. Biochem Biophys Res Commun 2004; 325: 1304-1311.

48. Ito J, Takita M, Takimoto K, Maturana AD. Enigma homolog 1 promotes myogenic gene expression and differentiation of C2C12 cells. Biochem Biophys Res Commun 2013; 435: 483-487.

49. Kuroda S, Tokunaga C, Kiyohara Y, Higuchi O, Konishi H, Mizuno K et al. Protein-protein interaction of zinc finger LIM domains with protein kinase C. J Biol Chem 1996; 271: 31029-31032.

50. Asp J, Thornemo M, Inerot S, Lindahl A. The helix-loop-helix transcription factors Id1 and Id3 have a functional role in control of cell division in human normal and neoplastic chondrocytes. FEBS Lett 1998; 438: 85-90.

51. Kurooka H, Yokota Y. Nucleo-cytoplasmic shuttling of Id2, a negative regulator of basic helixloop-helix transcription factors. J Biol Chem 2005; 280: 4313-4320.

52. Wang S, Sdrulla A, Johnson JE, Yokota Y, Barres BA. A role for the helix-loop-helix protein Id2 in the control of oligodendrocyte development. Neuron 2001; 29: 603-614.
53. Haraguchi T, Ozaki Y, Iba H. Vectors expressing efficient RNA decoys achieve the long-term suppression of specific microRNA activity in mammalian cells. Nucleic Acids Res 2009; 37 e43.

54. Qiu H, Zhong J, Luo L, Liu N, Kang K, Qu J et al. A PCR-based method to construct lentiviral vector expressing double tough decoy for miRNA inhibition. PLoS One 2015; 10: e0143864.

55. McCarthy JJ, Mula J, Miyazaki M, Erfani R, Garrison K, Farooqui AB et al. Effective fiber hypertrophy in satellite cell-depleted skeletal muscle. Development 2011; 138: 3657-3666.

56. Jalava $P$, Kuopio T, Juntti-Patinen L, Kotkansalo T, Kronqvist $P$, Collan Y. Ki67 immunohistochemistry: a valuable marker in prognostication but with a risk of misclassification: proliferation subgroups formed based on Ki67 immunoreactivity and standardized mitotic index. Histopathology 2006; 48: 674-682.

(1)(2) (2) This work is licensed under a Creative Commons Attribution-NonCommercial-ShareAlike 4.0 International License. The images or other third party material in this article are included in the article's Creative Commons license, unless indicated otherwise in the credit line; if the material is not included under the Creative Commons license, users will need to obtain permission from the license holder to reproduce the material. To view a copy of this license, visit http://creativecommons.org/licenses/by-nc-sa/4.0/

(C) The Author(s) 2016

Supplementary Information accompanies this paper on Cell Death and Differentiation website (http://www.nature.com/cdd) 